\title{
Lycorine attenuates lipopolysaccharide-induced acute lung injury through the HMGB1/TLRs/NF-KB pathway
}

\author{
Xin $\mathrm{Ge}^{1,2} \cdot$ Xianglin Meng $^{1} \cdot$ Dongsheng $\mathrm{Fei}^{1} \cdot \mathrm{Kai}^{\mathrm{Kang}}{ }^{1} \cdot$ Qiubo Wang $^{3} \cdot$ Mingyan Zhao $^{1} \mathbb{C}$
}

Received: 31 March 2020 / Accepted: 25 July 2020 / Published online: 1 August 2020

(c) King Abdulaziz City for Science and Technology 2020

\begin{abstract}
Lung injury associated with systemic inflammatory response is a common problem affecting human health. Previous studies have shown that lycorine exerts a anti-inflammatory effect. However, whether lycorine alleviates lung injury remains unclear. To explore this issue, BALB/c mice and MLE-12 cells were treated with lipopolysaccharide (LPS) to establish lung injury mouse model and cell model, respectively. Glycyrrhizic acid, known as an inhibitor of ALI, was also used to study the effects of lycorine in vitro. Our results showed that after LPS treatment, the lung injury score, lung wet-to-dry weight ratio, and malondialdehyde (MDA) production in the lung tissues and the expression levels of tumor necrosis factor- $\alpha$, interleukin- $1 \beta$, and interleukin-6 in bronchoalveolar lavage fluid were significantly increased, whereas their levels were decreased by lycorine. Additionally, LPS injection activated the high-mobility group box 1 (HMGB1)/Toll-like receptors (TLRs)/NF-kB pathway. However, lycorine treatment attenuated the activity of the HMGB1/TLRs/NF-kB pathway in the lung tissues. In vitro studies showed that lycorine administration significantly decreased the levels of inflammatory cytokines and MDA and attenuated the activity of the HMGB1/TLRs/NF-kB pathway in LPS-treated cells. Moreover, the inhibitory effects of lycorine on the inflammatory response and oxidative stress in LPS-treated lung cells were similar with that of glycyrrhizic acid, and this inhibition was intensified by both lycorine and glycyrrhizic acid treatment. We suggest that lycorine could alleviate LPSinduced lung injury of inflammation and oxidative stress by blocking the HMGB1/TLRs/NF-kB pathway, which gives a new perspective for ALI therapy to treat lycorine as a potential treatment clinically.
\end{abstract}

Keywords Acute lung injury $\cdot$ Lycorine $\cdot$ Inflammatory response $\cdot$ Oxidative stress $\cdot$ HMGB1/TLRs/NF-kB pathway

\section{Introduction}

Acute lung injury (ALI) is a clinical syndrome results from various direct or indirect injury factors, characterized by pulmonary edema, inflammatory responses, and surfactant dysfunction (Fan et al. 2018). ALI usually leads to further clinical outcomes, including acute respiratory distress

Mingyan Zhao

mingyan_hmu@aliyun.com

1 Department of ICU, The First Affiliated Hospital of Harbin Medical University, No. 23 Youzheng Street, Harbin 150001, Heilongjiang, People's Republic of China

2 Department of ICU, Wuxi 9th Affiliated Hospital of Soochow University, Wuxi 214000, Jiangsu, People's Republic of China

3 Department of Clinical Laboratory, Wuxi 9th Affiliated Hospital of Soochow University, Wuxi 214000, Jiangsu, People's Republic of China syndrome (ARDS) and hypoxemia (Fan et al. 2018; Fan and Fan 2018). Therefore, it is necessary to explore new therapeutic approaches and therapeutic targets for the treatment of ALI.

Imbalance in a variety of complex reactions, including inflammatory responses and oxidative stress, is the main cause of lung injury. Inflammatory responses involve the activation of inflammatory cells and the release of inflammatory cytokines (Park et al. 2001). In response to injury stimulation, neutrophils, macrophages, endothelial cells, and mast cells are activated and produce various cytotoxic substances, including pro-inflammatory factors, leading to lung injury and further acute hypoxic respiratory insufficiency or respiratory failure (Butt et al. 2016; Herold et al. 2013). Additionally, when the organisms are adversely stimulated, the oxidation and antioxidant processes become out of balance, resulting in the accumulation of peroxidation products, including reactive oxygen species (ROS) and malondialdehyde (MDA), and further cell death (Guo and Ward 2007). Moreover, the accumulation of 
peroxidation products is also associated with the activation of inflammatory responses. Specifically, ROS could regulate the expression levels of pro-inflammatory factors through the nuclear factor- $\mathrm{\kappa B}(\mathrm{NF}-\mathrm{\kappa B})$ pathway and chromatin remodeling (Rahman et al. 2004). It has been reported that the inhibition of inflammation response and oxidative stress effectively attenuated lipopolysaccharide (LPS)-induced ALI (Jiang et al. 2016; Lei et al. 2018). Mechanism studies have shown that this inhibition was achieved by blocking the toll-like receptor 4 (TLR4)/NF-kB pathway and nuclear factor E2-related factor 2 (Nrf2)/heme oxygenase-1 (HO-1) pathway (Deng et al. 2017; Lei et al. 2018). Collectively, inflammatory response and oxidative stress may be therapeutic targets for the treatment of lung injury.

Lycorine is a natural alkaloid isolated from lycoris and displays a variety of biological efficacy, including antitumor, anti-virus, and anti-malaria (Guo et al. 2016; Nair and van Staden 2019; Ying et al. 2017). Early studies showed that lycorine also had anti-inflammatory efficacy and improved the survival rate of mice with LPS-induced septic shock (Citoglu et al. 2012; Kang et al. 2012). However, whether lycorine alleviates LPS-mediated ALI needs further investigation. High-mobility group box 1 (HMGB1) is a key regulator of ALI. HMGB1 mediated the activation of the TLR4/NF- $\kappa B$ pathway and the blockade of TLR4/ NF- $\kappa B$ pathway relieved LPS-induced ALI (Meng et al. 2018). Moreover, the evidence revealed that lycorine could inhibit HMGB1 expression in cancer progression (Roy et al. 2016). Based on the above, we hypothesized that lycorine could alleviate LPS-induced ALI via blocking the HMGB1/ TLR4/NF-kB pathway.

In the present study, we found that LPS treatment triggered significant lung injury and induced inflammatory response and oxidative stress in the lung, which was attenuated by lycorine. Mechanism studies showed that the activation of HMGB1/TLRs/NF-KB pathway induced by LPS increased inflammatory responses and oxidative stress, while its blockade by lycorine effectively suppressed pulmonary inflammatory response and oxidative stress, and further alleviated lung injury.

\section{Materials and methods}

\section{Animal grouping and treatment}

Male BALB/c mice aged 8 weeks were adaptively fed for 1 week. All animals had free access to water and were housed at a temperature of $25 \pm 1{ }^{\circ} \mathrm{C}$ with a $12 \mathrm{~h}: 12 \mathrm{~h}$ light-dark cycle. The animals were randomly divided into four groups: control group, LPS group, LPS + lycorine $(20 \mathrm{mg} / \mathrm{kg})$ group, and LPS + lycorine $(40 \mathrm{mg} / \mathrm{kg})$ group.
For the establishment of ALI model, mice were instilled with LPS intratracheally ( $2 \mathrm{mg} / \mathrm{kg}$, dissolved in $50 \mu \mathrm{l}$ saline), while the control group was given the same amount of saline. For the treatment group, mice were received lycorine intraperitoneally ( $20 \mathrm{mg} / \mathrm{kg}$ or $40 \mathrm{mg} / \mathrm{kg}$ ) $1 \mathrm{~h}$ before LPS treatment. After $12 \mathrm{~h}$ of LPS treatment, all animals were sacrificed. Bronchoalveolar lavage fluid (BALF) and lung tissues were collected for subsequent experiments. LPS and lycorine were purchased from Aladdin Biochemical Technology Co., Ltd. (Shanghai, China).

\section{Hematoxylin-eosin (H\&E) staining}

The lung tissue samples were fixed with $10 \%$ formaldehyde, embedded with paraffin, and then sectioned. Lung sections $(5 \mu \mathrm{m})$ were stained with hematoxylin and eosin according to the manufacturer's instructions. Subsequently, the sections stained with hematoxylin and eosin were observed and photographed under a microscope (Olympus, Tokyo, Japan) (magnification, 200 $\times$ ). Blind lung injury scoring system was used to calculate the lung injury score. In short, alveolar congestion, alveolar wall thickening and edema, and alveolar cell infiltration scored 0-3 (0: None, 1: Mild, 2: Moderate, 3: Severe), with a maximum score of 9 (Toba et al. 2016).

\section{Lung wet-to-dry weight ratio}

Fresh lung tissues (left lung) were collected. After removing other tissues, the wet weight of lung tissues was measured. Then, the lungs were placed in an oven at $70{ }^{\circ} \mathrm{C}$ for $24 \mathrm{~h}$ to remove all moisture, and the dry weight was determined. Finally, the lung wet/dry weight ratio was calculated.

\section{Enzyme-linked immunosorbent (ELISA) assay}

The expression levels of tumor necrosis factor- $\alpha$ (TNF- $\alpha$ ), interleukin-1 $\beta$ (IL-1 $\beta$ ), and interleukin-6 (IL-6) in BALF and cells were detected using ELISA Kits (Boster, Wuhan, China). The optical density (OD) values at $450 \mathrm{~nm}$ and $570 \mathrm{~nm}$ were detected with a microplate reader (Biotek, Winooski, VT, USA).

\section{Malondialdehyde (MDA) assay}

The lung tissues were homogenized and centrifuged to obtain the supernatant. Radioimmunoprecipitation assay lysis buffer (Solarbio, Beijing, China) was used to lyse cells. The lysate was then centrifuged to obtain the supernatant. The protein concentration was measured using Bicinchoninic acid (BCA) protein concentration kit (Beyotime, Shanghai, China) and then diluted to $1 \mathrm{mg} / \mathrm{ml}$ with saline. Subsequently, the MDA detection kit (Jiancheng, Nanjing, 
China) was used to measure the MDA level according to the manufacturer's instructions.

\section{Immunohistochemistry assay}

The lung sections were incubated with $3 \% \mathrm{H}_{2} \mathrm{O}_{2}$ for 15 min at room temperature to eliminate endogenous peroxidase activity. After treated with 5\% goat serum for $15 \mathrm{~min}$ at room temperature, the sections were incubated with antiHMGB1 antibody (1:50, abclonal, Wuhan, China) at $4{ }^{\circ} \mathrm{C}$ overnight. Then, sections were washed with PBS for three times ( $5 \mathrm{~min} / \mathrm{time}$ ) and incubated with horseradish peroxidase (HRP)-labeled secondary antibodies (1:500, Thermo Fisher, Waltham, MA, USA) for $1 \mathrm{~h}$ at $37^{\circ} \mathrm{C}$. After washing three times with PBS ( $5 \mathrm{~min} /$ time), the sections were treated with 3,3 $\mathrm{N}$-diaminobenzidine tetrahydrochloride reagent $(100 \mu \mathrm{l})$ (Solarbio). Subsequently, hematoxylin (Solarbio) was counterstained for $3 \mathrm{~min}$. Finally, the slices were observed and photographed under a microscope (Olympus) (magnification, 200 $\times$ ).

\section{Western blot analysis}

Total proteins were extracted from lung tissues and cells. BCA protein concentration kit (Beyotime) was used to detect protein concentration. Equal amount $(20 \mu \mathrm{g}, 20 \mu \mathrm{l}$ per lane, $1 \mu \mathrm{g} /$ $\mu \mathrm{l})$ of protein was separately by $10 \%$ SDS-PAGE. After transferred onto polyvinylidene difluoride membranes, the proteins were incubated with primary antibodies at $4{ }^{\circ} \mathrm{C}$ overnight. Subsequently, the membranes were incubated with HRPconjugated anti-rabbit/mouse secondary antibodies (1:5000, Beyotime) for $2 \mathrm{~h}$ at room temperature. Then, the membranes were visualized with chemiluminescence reagent and the optical density values were analyzed by Gel-Pro-Analyzer software. $\beta$-actin was used as an internal control to standardize the target protein levels. The primary antibodies used in the present study were: anti-HMGB1 (1:1000, Proteintech, Wuhan, China), anti-TLR4 (1:1000, Proteintech), anti-TLR5 (1:1000, Proteintech), anti-Myd88 (1:1000, Affinity, Cincinnati, OH, USA), anti-P65/P-P65 (Ser 536) (1:1000, Affinity), anti-IкB $\alpha /$ $\mathrm{P}-\mathrm{I} \kappa \mathrm{B} \alpha($ Ser $32 / \mathrm{Ser} 36)$ (1:1000, Affinity), and anti- $\beta$-actin (1:1000, Santa Cruz Biotechnology, Santa Cruz, CA, USA).

\section{Quantitative real-time PCR (RT-qPCR)}

Total RNA was extracted from lung tissues and cells. With the help of Super M-MLV reverse transcriptase and RNase inhibitor, reverse transcription PCR was performed to obtain complementary DNA (cDNA). Subsequently, RT-qPCR was performed using cDNA, primers, $2 \times$ Power Taq PCR MasterMix, and SYBR Green. The expression levels of the target genes were normalized with $\beta$-actin. The primers (GenScrip, Nanjing, China) for RT-qPCR were as follows:
HMGB1 Forward: 5'...GGATATTGCTGCCTACAG AG...3'

Reverse: 5'... CTTCATCCTCCTCATCAT CT....3'

TLR4 Forward: 5'... AGCAGGTGGAATTGTATC GC....3'

Reverse: $5^{\prime} \ldots$ TCAGGTCCAAGTTGCCGT TT....3'

TLR5 Forward: $5^{\prime} \ldots$ CCCTTGTAGTCATAAAGT TCCG....3'

Reverse: 5'... AAGCATTCTGTGCCCATT CA...3'

$\beta$-Actin Forward: $5^{\prime} \ldots$ CTGTGCCCATCTACGAGG GCTAT.... $3^{\prime}$

Reverse: $5^{\prime}$... TTTGATGTCACGCACGAT TTCC....3'

\section{Cell culture and treatment}

Mouse lung epithelial (MLE)-12 cells were cultured in a Dulbecco's minimal essential medium/Ham's F12 medium (Zhongqiaoxinzhou Biotech, Shanghai, China) containing $10 \%$ fetal bovine serum in an incubator at $37{ }^{\circ} \mathrm{C}$ supplied with $5 \% \mathrm{CO}_{2}$. Cells were pretreated with lycorine (10 $\mu \mathrm{M}$, Aladdin, Shanghai, China) or/and glycyrrhizic acid ( $1 \mathrm{mmol} / \mathrm{L}$, MedChem Express, Monmouth Junction, NJ, USA) for $3 \mathrm{~h}$ and then treated with LPS $(2 \mu \mathrm{g} / \mathrm{mL}$, Sigma, St. Louis, MO, USA) for $6 \mathrm{~h}$.

\section{Immunofluorescence staining}

Lung sections were fixed in $4 \%$ paraformaldehyde for $15 \mathrm{~min}$ and then incubated with $0.1 \%$ Triton X-100 for 30 min. After washing three times with PBS (5 min/time), the sections were blocked with goat serum for $15 \mathrm{~min}$. Subsequently, sections were incubated with anti-HMGB1 antibody (1:50, abclonal, Wuhan, China) at $4{ }^{\circ} \mathrm{C}$ overnight, followed by $\mathrm{Cy} 3$ labeled goat anti-rabbit secondary antibody (1: 200, Beyotime). After washing three times (5 min/time) with PBS, the sections were treated with 4',6-diamidino-2-phenylindole (Aladdin) for $30 \mathrm{~min}$. Finally, sections were observed and photographed (Olympus) (magnification, 400 ×).

\section{Statistical analysis}

All data were mean \pm standard deviation. Graphpad Prism 8.0 was used for data analysis. Differences between multiple groups were analyzed by one-way ANOVA. $P<0.05$ was considered statistically significant. 


\section{Results}

\section{Lycorine alleviated LPS-induced lung injury, inflammatory response, and oxidative stress in mice.}

We first investigated the effects of lycorine on LPS-induced lung injury. The results showed that compared with control group, LPS treatment resulted in pathological damage, including alveolar congestion, alveolar wall thickening, and edema (Fig. 1a). The lung injury score in LPS group was significantly elevated as compared with control group (Fig. 1a, $P<0.05$ ). However, compared with LPS group, the severity of alveolar congestion, alveolar wall thickening, and edema in LPS + lycorine groups was decreased (Fig. 1a). The lung injury score in LPS + lycorine groups was lower than that in LPS group (Fig. 1a, $P<0.05$ ). As shown in Fig. 1b, compared with control group, the lung wet-to-dry weight ratio was significantly increased after LPS challenged $(P<0.05)$. However, the lung wet-to-dry weight ratio in LPS + lycorine $(40 \mathrm{mg} / \mathrm{kg})$ group was significantly decreased compared with LPS group (Fig. 1b, $P<0.05$ ). LPS treatment resulted in the accumulation (a)
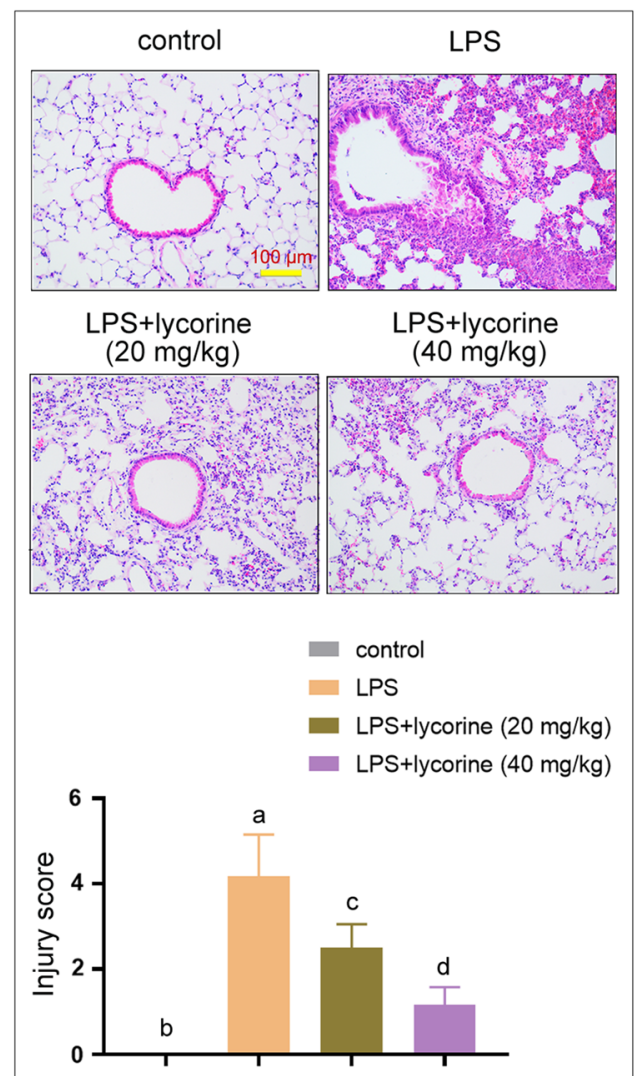

(b)

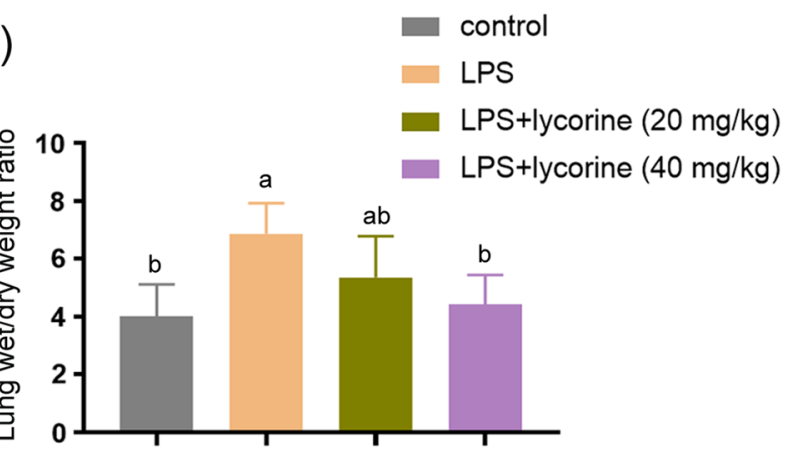

(c)

(d)
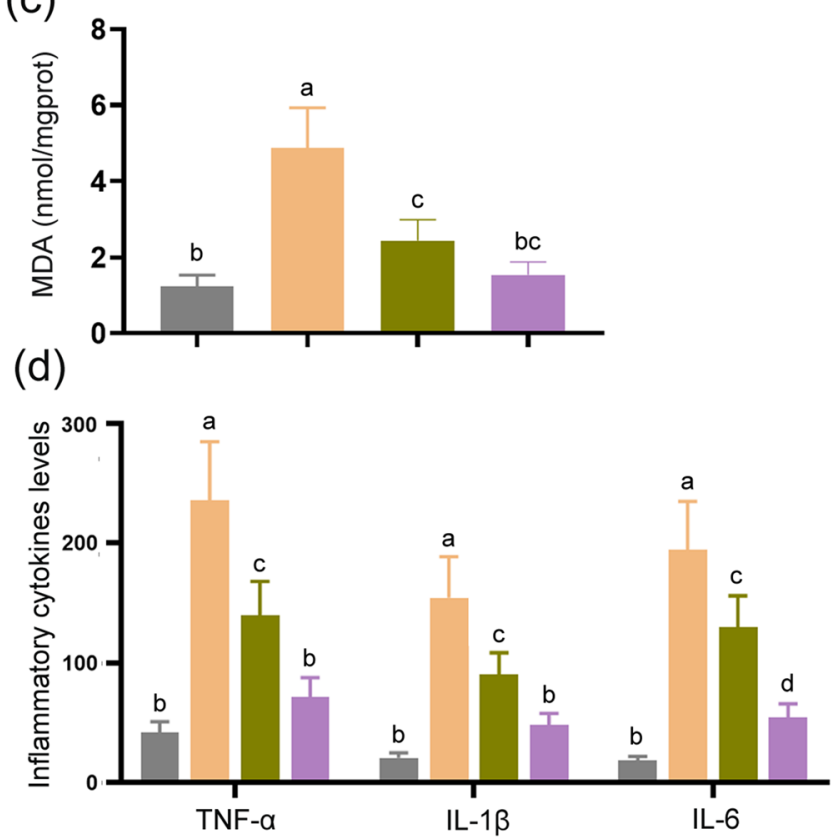

Fig. 1 Lycorine alleviated LPS-induced lung injury, inflammatory response, and oxidative stress in mice. One hour before challenged with LPS $(2 \mathrm{mg} / \mathrm{kg}$, dissolved in $50 \mu \mathrm{l}$ saline) for $12 \mathrm{~h}, \mathrm{BALB} / \mathrm{c}$ mice were treated with or without lycorine $(20 \mathrm{mg} / \mathrm{kg}$ or $40 \mathrm{mg} / \mathrm{kg})$. 1, control group; 2, LPS group; 3, LPS + lycorine $(20 \mathrm{mg} / \mathrm{kg}$ ) group; 4, LPS + lycorine (40 mg/kg) group. a Representative histological images of lung lesions and the lung injury score were obtained by Hematoxylin-Eosin staining, Scale bar $=100 \mu \mathrm{m}$. b, c The lung wet/dry weight ratio and the levels of MDA in the lung tissues were measured in each group. d The expression levels of TNF- $\alpha$, IL-1 $\beta$, and IL-6 in each group were measured by enzyme-linked immunosorbent assay. $N=6$. Data are means $\pm \mathrm{SD}$. A superscript with the same letter (such as a and ab) means that the difference is not significant, and a superscript with different letter (such as a and b) means that the difference is significant $(P<0.05)$. LPS lipopolysaccharide, $M D A$ malondialdehyde, $T N F-\alpha$ tumor necrosis factor- $\alpha, I L-1 \beta$ interleukin- $1 \beta, I L-6$ interleukin-6 
of MDA in the lung issues, which was decreased by lycorine (Fig. 1c, $P<0.05$ ). Additionally, the expression levels of pro-inflammatory factors in BALF were detected by ELISA assay. As shown in Fig. 1d, the expression levels of TNF- $\alpha$, IL-1 $\beta$, and IL-6 in LPS group were significantly up-regulated as compared with control group, whereas lycorine treatment inhibited LPS-induced TNF- $\alpha$, IL-1 $\beta$, and IL- 6 elevation in a dose-dependent manner in BALF $(P<0.05)$. Therefore, the results suggested that LPS treatment successfully induced severe lung injury in the lungs accompanied by pulmonary edema, inflammatory response, and oxidative stress, which was ameliorated by lycorine.

\section{Lycorine blocked HMGB1/TLRs/NF-kB pathway in LPS-treated mice.}

We further explored the mechanism of lycorine in alleviating LPS-induced lung injury. As shown in Fig. 2a, the expression level of HMGB1 measured by immunohistochemistry assay in LPS group was higher than that in control group, while lycorine treatment inhibited the production of HMGB 1 induced by LPS. Furthermore, compared to the control group, the protein levels of HMGB1, TLR4, TLR5, Myd88, P-P65, and P-IкB $\alpha$ in LPS group were observably increased and I $\mathrm{B} \alpha$ protein level in LPS group was significantly decreased $(P<0.05)$. However, compared with LPS group, the protein levels of HMGB1, TLR4, TLR5, Myd88, P-P65, and P-IкB $\alpha$ in LPS + lycorine groups were markedly decreased and IкB $\alpha$ level was increased (Fig. $2 b, P<0.05$ ). Consistently, the mRNA expression levels of HMGB1, TLR4, and TLR5 were markedly increased in LPS group as compared with control, whereas their levels were significantly decreased in LPS + lycorine groups as compared with LPS group (Fig. 2c, $P<0.05$ ). Therefore, these results indicated that lycorine inhibited LPS-induced activation of the HMGB1/TLRs/NF-kB pathway.

\section{Lycorine alleviated LPS-induced inflammatory response and oxidative stress in MLE-12 cells}

We also established a cell model with LPS. The results showed that compared with control group, the TNF- $\alpha$, IL- $1 \beta$, IL-6, and MDA levels in LPS group were significantly increased, while their levels in LPS + lycorine and LPS + glycyrrhizic acid groups were decreased as compared with LPS group, respectively (Fig. 3a-d, $P<0.05$ ). Moreover, the inhibitory effect of lycorine on the expression levels of pro-inflammatory factors was enhanced by glycyrrhizic acid (Fig. 3a-d, $P<0.05$ ). Overall, these results indicated that lycorine inhibits LPS-induced inflammatory response and oxidative stress, which is consistent with in vivo studies. Moreover, the combined effect of lycorine and glycyrrhizic acid is more conducive to relieving LPS-induced lung injury.

\section{Lycorine blocked HMGB1/TLRs/NF-kB pathway in LPS-treated MLE-12 cells}

As presented in Fig. 4a, LPS treatment increased HMGB1 expression level as compared with control group. However, lycorine treatment decreased HMGB1 expression level as compared with the LPS group, and the decrease was further enhanced by glycyrrhizic acid (Fig. 4a). Moreover, compared with control group, LPS triggered HMGB1, TLR4, TLR5, Myd88, P-P65, and P-IкB $\alpha$ elevation, and decreased I $\mathrm{B} \alpha$ level $(P<0.05)$. However, lycorine and glycyrrhizic acid administration significantly inhibited the accumulation of HMGB1, TLR4, TLR5, Myd88, P-P65, and P-IкB $\alpha$ in LPS-treated cells (Fig. 4b, $P<0.05$ ). The trend of HMGB1, TLR4, and TLR5 mRNA levels in each group was consistent with their protein expression (Fig. $4 \mathrm{c}, P<0.05$ ). Furthermore, the inhibitory effect of lycorine on the HMGB1/ TLRs/NF- $\kappa$ B pathway was enhanced by glycyrrhizic acid (Fig. 4a-c, $P<0.05$ ). Overall, lycorine and glycyrrhizic acid can inhibit the activation of the LPS-induced HMGB1/ TLRs/NF- $\kappa$ B pathway. Co-treatment of lycorine and glycyrrhizic acid enhances this inhibitory effect.

\section{Discussion}

In the present study, we revealed the important role of lycorine in alleviating LPS-induced ALI. Increasing evidence suggested that inflammatory response is a vital cause of lung injury. Severe inflammatory response and overexpression of inflammatory factors IL- $1 \beta$, TNF- $\alpha$, IL-8, and IL-6 were observed in both lung injury animal models and patients with ARDS (Dong and Yuan 2018; Meduri et al. 1995). Additionally, studies showed that highly expressed inflammatory factors were also associated with poor prognosis of patients with ARDS, including IL-6 (Bouros et al. 2004; Meduri et al. 1995). The present study showed that LPStreated mice exhibited inflammatory response and overexpression of TNF- $\alpha$, IL- $1 \beta$, and IL- 6 , which is consistent with the previous results (Deng et al. 2017). In addition, oxidative stress is considered to be another important cause of lung damage. It can not only directly induce tissue damage, but also regulate pathways involved in pulmonary inflammatory response (Crapo 2003; Guo and Ward 2007). Severe oxidative stress was observed in both lung injury patients and animal models, and the MDA level was significantly elevated (Lei et al. 2018; Winterbourn et al. 2000). Consistent with the previous studies, the present study showed that LPS treatment resulted in the accumulation of oxidative stress and MDA in the lungs and cells, indicating that inflammatory response and oxidative stress play a crucial role in the induction of lung injury. It has been reported that drugs that inhibit inflammation and oxidation effectively attenuated 
(a)

(b)
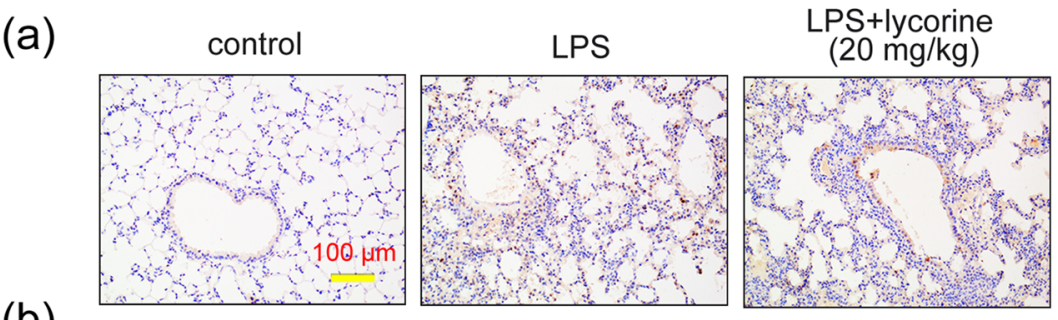

LPS+lycorine
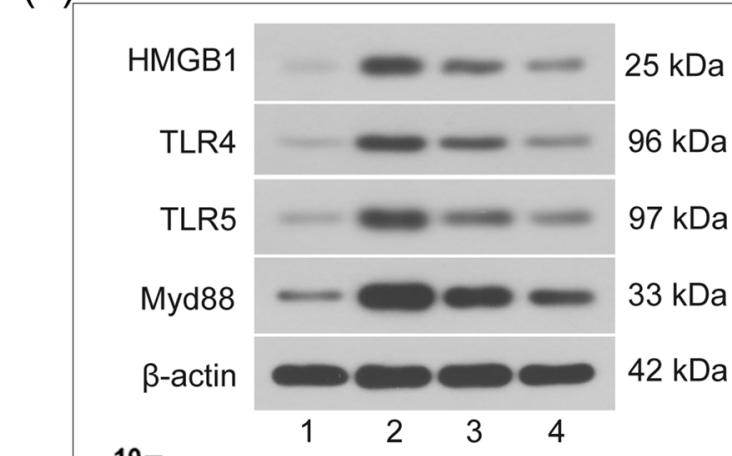

1 control 2 LPS

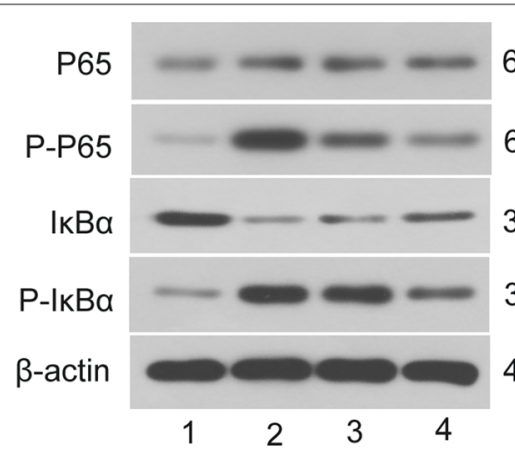

$65 \mathrm{kDa}$

$65 \mathrm{kDa}$

$39 \mathrm{kDa}$

3 LPS+lycorine $(20 \mathrm{mg} / \mathrm{kg})$

4 LPS+lycorine $(40 \mathrm{mg} / \mathrm{kg}$ )

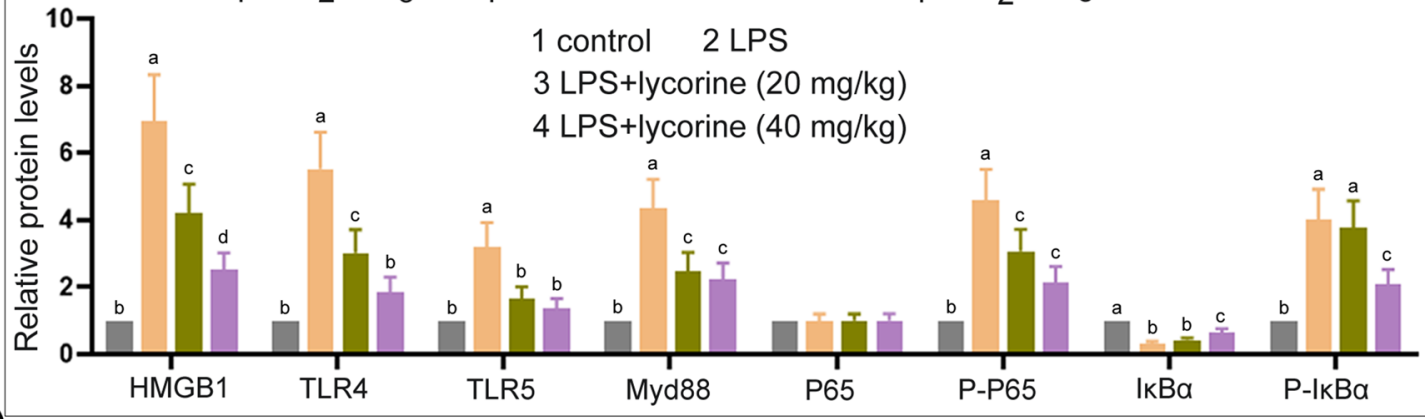

(c)

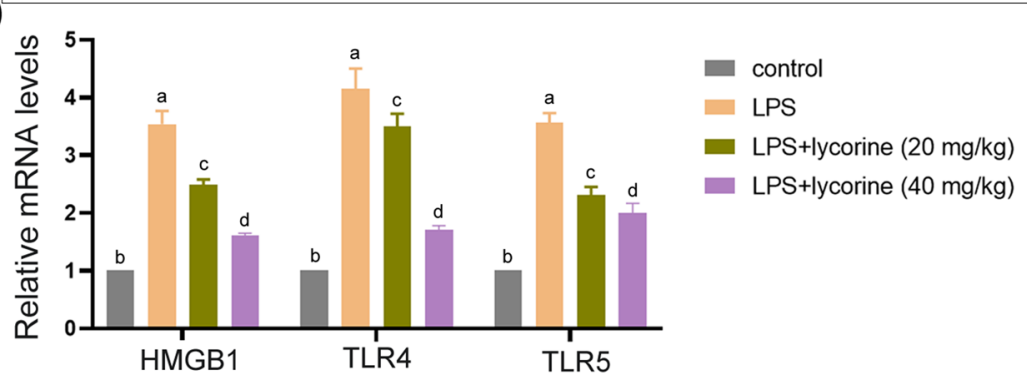

Fig. 2 Lycorine blocked HMGB1/TLRs/NF- $\kappa$ B pathway in LPStreated mice. One hour before challenged with LPS $(2 \mathrm{mg} / \mathrm{kg}$, dissolved in $50 \mu \mathrm{l}$ saline) for $12 \mathrm{~h}, \mathrm{BALB} / \mathrm{c}$ mice were treated with or without lycorine $(20 \mathrm{mg} / \mathrm{kg}$ or $40 \mathrm{mg} / \mathrm{kg})$. 1, control group; 2, LPS group; 3, LPS + lycorine (20 mg/kg) group; 4, LPS + lycorine $(40 \mathrm{mg} /$ $\mathrm{kg}$ ) group. a The expression of HMGB1 in the lung tissues was measured by immunohistochemistry assay, Scale bar $=100 \mu \mathrm{m}$. b Relative protein expression levels of HMGB1, TLR4, TLR5, Myc88, $\mathrm{P} 65, \mathrm{P}-\mathrm{P} 65, \mathrm{I} \kappa \mathrm{B} \alpha$, and $\mathrm{P}-\mathrm{I} \kappa \mathrm{B} \alpha$ in the lung tissues were determined

lung damage, including astaxanthin, cordycepin, and linarin (Bi et al. 2017; Han et al. 2018; Lei et al. 2018). Lycorine has been reported to be an effective inhibitor of tumor development and also had many other benefits, including reducing LPS-induced bone loss in mice and inhibiting intervertebral disc degeneration (Park et al. 2019; Wang et al. 2018). Here, we found that lycorine alleviated lung injury and inhibited by Western blot analysis. $\beta$-actin as an internal control. c The mRNA expression levels of HMGB1, TLR4, and TLR5 in each group were measured by RT-qPCR. $N=6$. Data are means \pm SD. A superscript with the same letter (such as a and ab) means that the difference is not significant, and a superscript with different letter (such as a and b) means that the difference is significant $(P<0.05)$. LPS lipopolysaccharide, HMGB1 the high-mobility group box 1, TLR Toll-like receptor, $R T-q P C R$ quantitative real-time PCR

pulmonary edema, inflammatory response, and oxidative stress. The results suggested that lycorine may be a potential treatment for ALI.

HMGB 1 is an inflammatory regulator related to sepsis and immune diseases, which is highly expressed in inflammatory diseases (Qu et al. 2019a). HMGB1 is the upstream regulator of TLR4 (Di Candia et al. 2017). In LPS-induced 


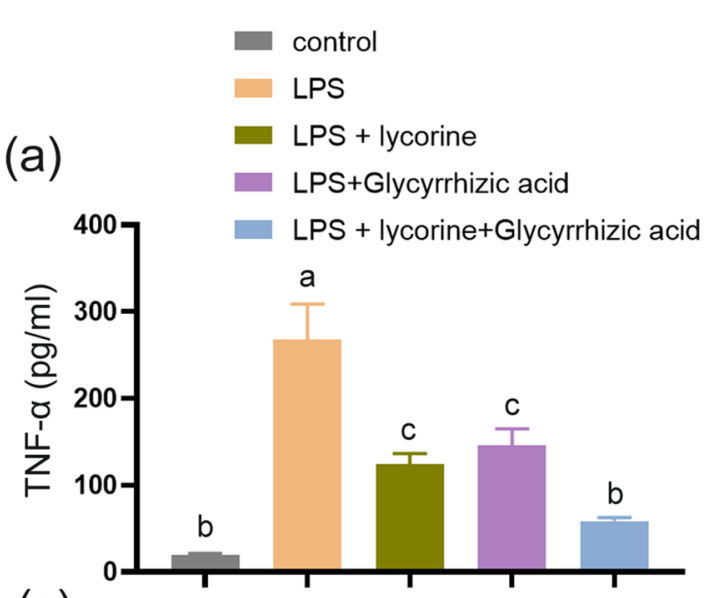

(c)

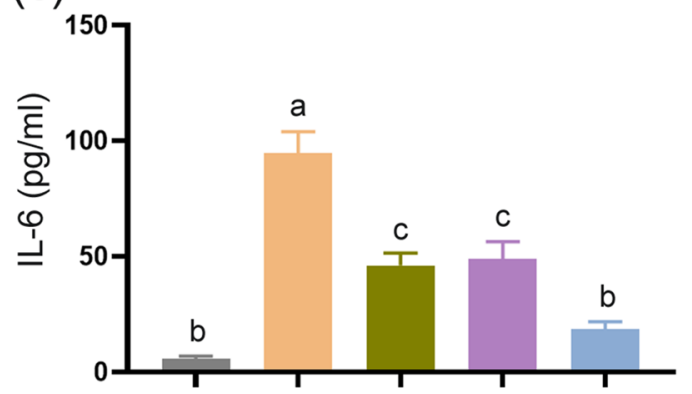

Fig. 3 Lycorine alleviated LPS-induced inflammatory response and oxidative stress in MLE-12 cells. MLE-12 cells were pretreated with lycorine $(10 \mu \mathrm{M})$ or/and glycyrrhizic acid $(1 \mathrm{mmol} / \mathrm{L})$ for $3 \mathrm{~h}$, and then treated with LPS $(2 \mu \mathrm{g} / \mathrm{mL})$ for $6 \mathrm{~h}$. 1, control group; 2, LPS group; 3, LPS + lycorine group; 4, LPS + glycyrrhizic acid group; 5, LPS + lycorine + glycyrrhizic acid group. a-c The expression levels of TNF- $\alpha$, IL-1 $\beta$, and IL- 6 were assessed by enzyme-linked immuno-

lung injury, HMGB1 mediated activation of the TLR4/ NF- $\mathrm{KB}$ signaling pathway, while HMGB1 down-regulation significantly blocked the TLR4/NF-kB pathway (Meng et al. 2018). Mechanism studies have shown that upon stimulation, phosphorylated I $\kappa \mathrm{B} \alpha$ dissociates from NF- $\kappa \mathrm{B}$ dimer (p65:p50), which is subsequently degraded by proteasomes. Activated NF- $\mathrm{\kappa B}$ is transferred from the cytoplasm to the nucleus to bind to the $\mathrm{\kappa B}$ site in the target gene promoter or enhancer, and then, P65 is phosphorylated by protein kinase to initiate the transcription of inflammatory factors and oxidative stress-related factors (Ghosh and Hayden 2008; Giridharan and Srinivasan 2018). Thus, the NF-kB pathway is critical for the induction of inflammatory response and oxidative stress. Additionally, the early studies showed that the blockade of TLR4/NF- $\kappa B$ pathway effectively inhibited oxidative stress and inflammatory responses in the lungs (Deng et al. 2017; Zhang et al. 2019). Therefore, the HMGB1/ TLR4/NF- $\kappa B$ pathway is an important pathway to induce inflammatory response and oxidative stress. In addition, TLR5 has been reported to be highly expressed in inflammatory diseases and tissues, and TLR5 knockdown significantly (b)

(d)
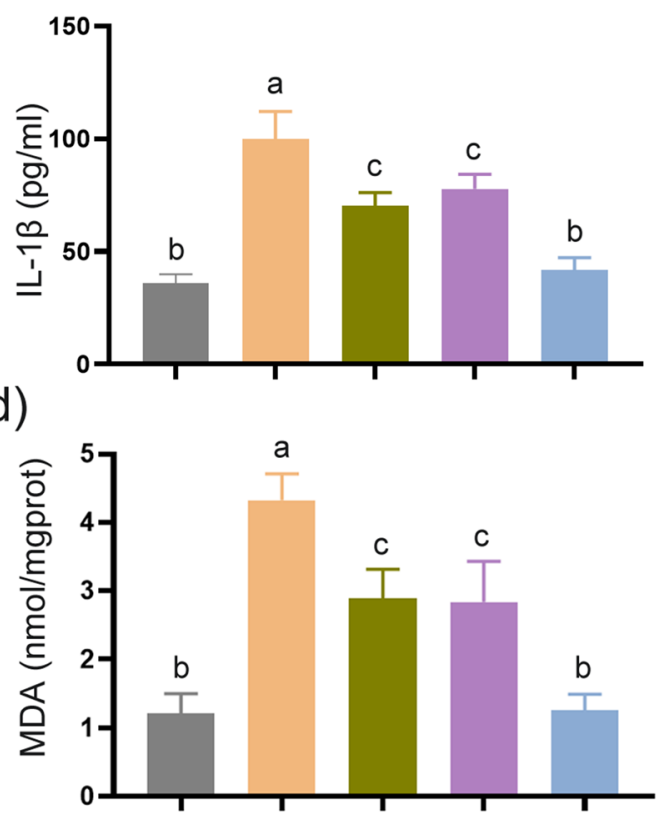

sorbent assay in MLE-12 cells in each group. $\mathbf{d}$ The MDA levels were measured in all the groups. $N=3$, data are means \pm SD. A superscript with the same letter (such as a and ab) means that the difference is not significant, and a superscript with different letter (such as a and b) means that the difference is significant $(P<0.05)$. LPS lipopolysaccharide, $M D A$ malondialdehyde, $T N F-\alpha$ tumor necrosis factor- $\alpha, I L$ $1 \beta$ interleukin- $1 \beta, I L-6$ interleukin-6

decreased the release of inflammatory cytokines (Gao et al. 2019; Ito et al. 2019). Moreover, TLR5 could activate NF-кB pathway (Yan et al. 2019). Here, we found that LPS treatment up-regulated HMGB1, TLR4, TLR5, Myd88, P-P65, and P-IкB $\alpha$ expression levels, whereas the up-regulation was suppressed by lycorine. The results suggested that lycorine may inhibit LPS-induced inflammatory response and oxidative stress via HMGB1/TLRs/NF-кB pathway. Glycyrrhizic acid is the main component of licorice. It is well known that glycyrrhizic acid has a positive effect on anti-inflammatory treatment (Yang et al. 2017). Glycyrrhizic acid is identified as an inhibitor of HMGB1. Studies showed that glycyrrhizic acid inhibited oxidative stress and inflammatory response in lung injury by inhibiting HMGB1 expression $(\mathrm{Qu}$ et al. 2019b; Zhao et al. 2016). Here, we found that lycorine has similar regulatory mechanisms with glycyrrhizic acid in alleviating lung injury. In addition, the synergistic effect of glycyrrhizic acid and lycorine is more conducive to the improvement of lung injury, which may provide a new idea for the treatment of lung injury. 


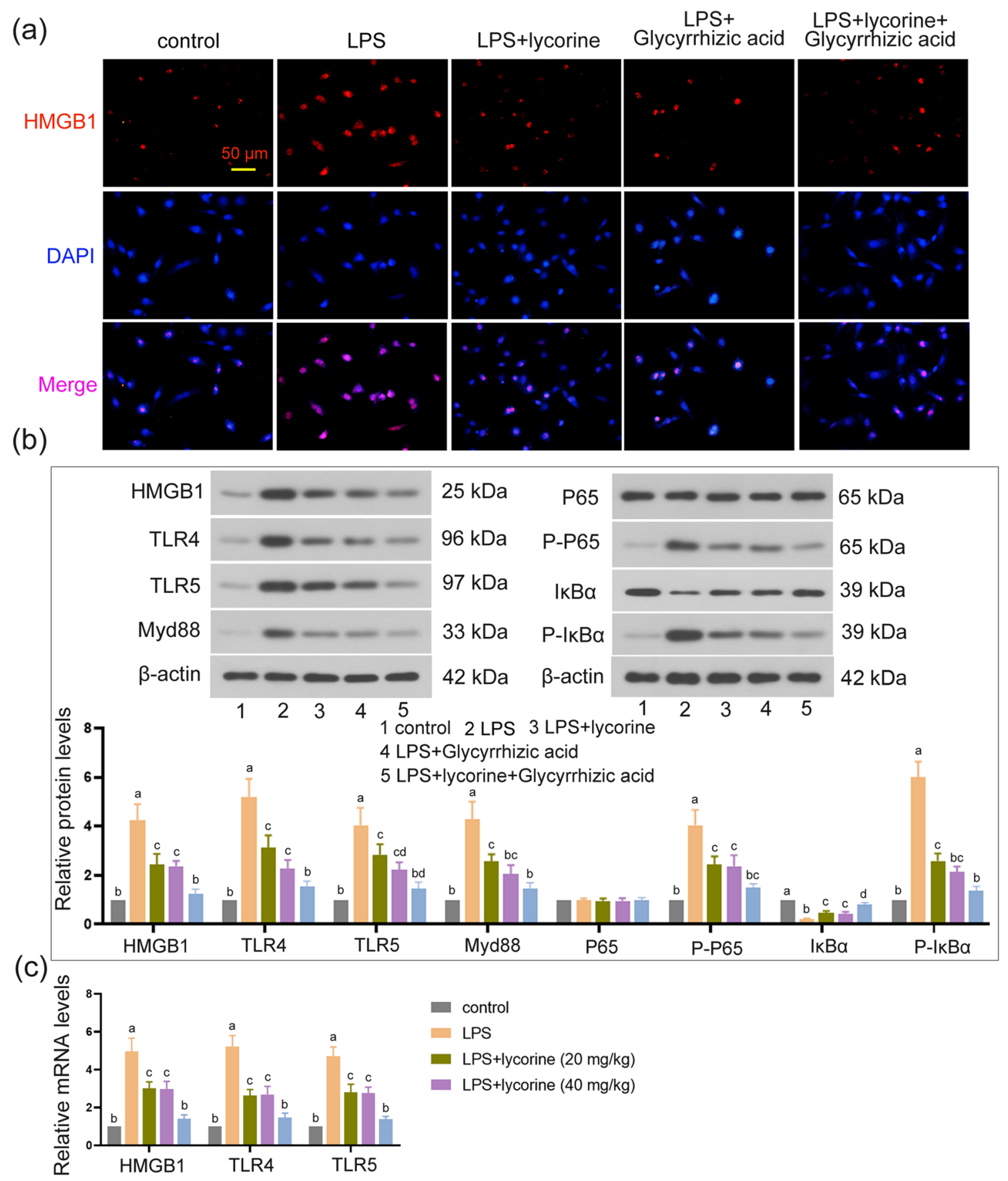

Fig. 4 Lycorine blocked HMGB1/TLRs/NF- $\mathrm{BB}$ pathway in LPStreated MLE-12 cells. MLE-12 cells were pretreated with lycorine $(10 \mu \mathrm{M})$ or/and glycyrrhizic acid $(1 \mathrm{mmol} / \mathrm{L})$ for $3 \mathrm{~h}$, and then treated with LPS $(2 \mu \mathrm{g} / \mathrm{mL})$ for $6 \mathrm{~h}$. 1, control group; 2, LPS group; 3, LPS + lycorine group; 4, LPS + glycyrrhizic acid group; 5, LPS + lycorine + glycyrrhizic acid group. a The expression of HMGB1 detected by immunofluorescence staining in each group. Scale bar $=50 \mu \mathrm{m}$. Red represents HMGB1 staining and blue for nuclei identification. b The protein expression levels of HMGB1,
TLR4, TLR5, Myc88, P65, P-P65, I $\kappa \mathrm{B} \alpha$, and $\mathrm{P}-\mathrm{I} \kappa \mathrm{B} \alpha$ in all groups were normalized to the level of $\beta$-actin. $\mathbf{c}$ The mRNA expression levels of HMGB1, TLR4, and TLR5 in each group were measured by RT-qPCR. $N=3$, data are means \pm SD. A superscript with the same letter (such as a and $a b$ ) means that the difference is not significant, and a superscript with different letter (such as a and b) means that the difference is significant $(P<0.05)$. LPS lipopolysaccharide, $H M G B 1$ the high-mobility group box $1, T L R$ toll-like receptor, $R T$ $q P C R$ quantitative real-time PCR 
Taken together, our results provide evidence that LPS can trigger lung injury accompanied by increased pulmonary edema, inflammatory response, and oxidative stress, which is attenuated by lycorine. Moreover, we further illustrate that lycorine may alleviate LPS-induced lung injury via blocking HMGB1/TLRs/NF-кB pathway.

Acknowledgements This study was supported by grants from the National Natural Scientific Foundation of China (no. 81772045).

\section{Compliance with ethical standards}

Conflict of interest The authors declare that they have no conflicts of interest.

Ethics approval All animal experiments were performed according to the National Institutes of Health guide for the care and use of laboratory animals and with the approval of the ethic committee of Harbin Medical University.

\section{References}

Bi J, Cui R, Li Z, Liu C, Zhang J (2017) Astaxanthin alleviated acute lung injury by inhibiting oxidative/nitrative stress and the inflammatory response in mice. Biomed Pharmacother 95:974-982. https://doi.org/10.1016/j.biopha.2017.09.012

Bouros D et al (2004) The clinical significance of serum and bronchoalveolar lavage inflammatory cytokines in patients at risk for Acute Respiratory Distress Syndrome. BMC Pulm Med 4:6. https://doi. org/10.1186/1471-2466-4-6

Butt Y, Kurdowska A, Allen TC (2016) Acute lung injury: a clinical and molecular review. Arch Pathol Lab Med 140:345-350. https ://doi.org/10.5858/arpa.2015-0519-RA

Citoglu GS, Acikara OB, Yilmaz BS, Ozbek H (2012) Evaluation of analgesic, anti-inflammatory and hepatoprotective effects of lycorine from Sternbergia fisheriana (Herbert) Rupr. Fitoterapia 83:81-87. https://doi.org/10.1016/j.fitote.2011.09.008

Crapo JD (2003) Oxidative stress as an initiator of cytokine release and cell damage. Eur Respir J Suppl 44:4s-6s. https://doi. org/10.1183/09031936.03.00000203a

Deng G et al (2017) Lianqinjiedu decoction attenuates LPS-induced inflammation and acute lung injury in rats via TLR4/NF-kappaB pathway. Biomed Pharmacother 96:148-152. https://doi. org/10.1016/j.biopha.2017.09.094

Di Candia L et al (2017) HMGB1 is upregulated in the airways in asthma and potentiates airway smooth muscle contraction via TLR4. J Allergy Clin Immunol 140:584 e588-587 e588. https:// doi.org/10.1016/j.jaci.2016.11.049

Dong Z, Yuan Y (2018) Accelerated inflammation and oxidative stress induced by LPS in acute lung injury: iotanhibition by ST1926. Int J Mol Med 41:3405-3421. https://doi.org/10.3892/ ijmm.2018.3574

Fan E, Brodie D, Slutsky AS (2018) Acute respiratory distress syndrome: advances in diagnosis and treatment. JAMA 319:698-710. https://doi.org/10.1001/jama.2017.21907

Fan EKY, Fan J (2018) Regulation of alveolar macrophage death in acute lung inflammation. Respir Res 19:50. https://doi. org/10.1186/s12931-018-0756-5

Gao F, Chen R, Xi Y, Zhao Q, Gao H (2019) Long noncoding RNA MALAT1 regulates sepsis in patients with burns by modulating
miR214 with TLR5. Mol Med Rep 19:3756-3766. https://doi. org/10.3892/mmr.2019.10028

Ghosh S, Hayden MS (2008) New regulators of NF-kappaB in inflammation. Nat Rev Immunol 8:837-848. https://doi.org/10.1038/ nri2423

Giridharan S, Srinivasan M (2018) Mechanisms of NF-kappaB p65 and strategies for therapeutic manipulation. J Inflamm Res 11:407419. https://doi.org/10.2147/JIR.S140188

Guo RF, Ward PA (2007) Role of oxidants in lung injury during sepsis. Antioxid Redox Signal 9:1991-2002. https://doi.org/10.1089/ ars.2007.1785

Guo Y et al (2016) A conserved inhibitory mechanism of a lycorine derivative against enterovirus and hepatitis $\mathrm{C}$ virus. Antimicrob Agents Chemother 60:913-924. https://doi.org/10.1128/ AAC.02274-15

Han X, Wu YC, Meng M, Sun QS, Gao SM, Sun H (2018) Linarin prevents LPSinduced acute lung injury by suppressing oxidative stress and inflammation via inhibition of TXNIP/NLRP3 and NFkappaB pathways. Int J Mol Med 42:1460-1472. https://doi. org/10.3892/ijmm.2018.3710

Herold S, Gabrielli NM, Vadasz I (2013) Novel concepts of acute lung injury and alveolar-capillary barrier dysfunction. Am J Physiol Lung Cell Mol Physiol 305:L665-681. https://doi.org/10.1152/ ajplung.00232.2013

Ito $\mathrm{H}$ et al (2019) Role of TLR5 in inflammation and tissue damage after intestinal ischemia-reperfusion injury. Biochem Biophys Res Commun 519:15-22. https://doi.org/10.1016/j.bbrc.2019.08.083

Jiang W et al (2016) The protective effect of Trillin LPS-induced acute lung injury by the regulations of inflammation and oxidative state. Chem Biol Interact 243:127-134. https://doi.org/10.1016/j. cbi.2015.09.010

Kang J et al (2012) Lycorine inhibits lipopolysaccharide-induced iNOS and COX-2 up-regulation in RAW264.7 cells through suppressing P38 and STATs activation and increases the survival rate of mice after LPS challenge. Int immunopharmacol 12:249-256. https:// doi.org/10.1016/j.intimp.2011.11.018

Lei J, Wei Y, Song P, Li Y, Zhang T, Feng Q, Xu G (2018) Cordycepin inhibits LPS-induced acute lung injury by inhibiting inflammation and oxidative stress. Eur J Pharmacol 818:110-114. https://doi. org/10.1016/j.ejphar.2017.10.029

Meduri GU, Headley S, Kohler G, Stentz F, Tolley E, Umberger R, Leeper K (1995) Persistent elevation of inflammatory cytokines predicts a poor outcome in ARDS. Plasma IL-1 beta and IL-6 levels are consistent and efficient predictors of outcome over time. Chest 107:1062-1073. https://doi.org/10.1378/chest.107.4.1062

Meng L et al (2018) The protective effect of dexmedetomidine on LPSinduced acute lung injury through the HMGB1-mediated TLR4/ NF-kappaB and PI3K/Akt/mTOR pathways. Mol Immunol 94:717. https://doi.org/10.1016/j.molimm.2017.12.008

Nair JJ, van Staden J (2019) Antiplasmodial lycorane alkaloid principles of the plant family amaryllidaceae. Planta Med 85:637-647. https://doi.org/10.1055/a-0880-5414

Park HJ, Gholam-Zadeh M, Suh JH, Choi HS (2019) Lycorine attenuates autophagy in osteoclasts via an axis of mROS/TRPML1/ TFEB to Reduce LPS-induced bone loss. Oxid Med Cell Longev 2019:8982147. https://doi.org/10.1155/2019/8982147

Park WY et al (2001) Cytokine balance in the lungs of patients with acute respiratory distress syndrome. Am J Respir Crit Care Med 164:1896-1903. https://doi.org/10.1164/ajrccm.164.10.2104013

Qu L et al (2019a) High-mobility group box 1 (HMGB1) and autophagy in acute lung injury (ALI): a review. Med Sci Monit 25:1828-1837. https://doi.org/10.12659/MSM.912867

Qu L et al (2019b) Glycyrrhizic acid ameliorates LPS-induced acute lung injury by regulating autophagy through the PI3K/AKT/ mTOR pathway. Am J Transl Res 11:2042-2055

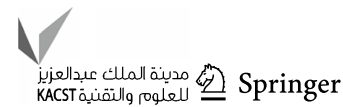


Rahman I, Marwick J, Kirkham P (2004) Redox modulation of chromatin remodeling: impact on histone acetylation and deacetylation NF-kappaB and pro-inflammatory gene expression. Biochem Pharmacol 68:1255-1267. https://doi.org/10.1016/j. bcp.2004.05.042

Roy M et al (2016) Lycorine downregulates HMGB1 to inhibit autophagy and enhances bortezomib activity in multiple myeloma. Theranostics 6:2209-2224. https://doi.org/10.7150/thno.15584

Toba H et al (2016) XB130 deficiency enhances lipopolysaccharide-induced septic response and acute lung injury. Oncotarget 7:25420-25431. https://doi.org/10.18632/oncotarget.8326

Wang G et al (2018) Lycorine suppresses endplate-chondrocyte degeneration and prevents intervertebral disc degeneration by inhibiting NF-kappaB signalling pathway. Cell Physiol Biochem 45:12521269. https://doi.org/10.1159/000487457

Winterbourn CC, Buss IH, Chan TP, Plank LD, Clark MA, Windsor JA (2000) Protein carbonyl measurements show evidence of early oxidative stress in critically ill patients. Crit Care Med 28:143149. https://doi.org/10.1097/00003246-200001000-00024

Yan J, Shen S, He Y, Li Z (2019) TLR5 silencing reduced hyperammonaemia-induced liver injury by inhibiting oxidative stress and inflammation responses via inactivating NF-kappaB and MAPK signals. Chem Biol Interact 299:102-110. https://doi. org/10.1016/j.cbi.2018.11.026

Yang R, Yuan BC, Ma YS, Zhou S, Liu Y (2017) The anti-inflammatory activity of licorice, a widely used Chinese herb. Pharm Biol 55:5-18. https://doi.org/10.1080/13880209.2016.1225775

Ying X, Huang A, Xing Y, Lan L, Yi Z, He P (2017) Lycorine inhibits breast cancer growth and metastasis via inducing apoptosis and blocking Src/FAK-involved pathway. Sci China Life Sci 60:417428. https://doi.org/10.1007/s11427-016-0368-y

Zhang ZM, Wang YC, Chen L, Li Z (2019) Protective effects of the suppressed NF-kappaB/TLR4 signaling pathway on oxidative stress of lung tissue in rat with acute lung injury. Kaohsiung $\mathrm{J}$ Med Sci 35:265-276. https://doi.org/10.1002/kjm2.12065

Zhao H, Zhao M, Wang Y, Li F, Zhang Z (2016) Glycyrrhizic acid prevents sepsis-induced acute lung injury and mortality in rats. $\mathrm{J}$ Histochem Cytochem 64:125-137. https://doi.org/10.1369/00221 55415610168 